Conclusion We have identified a subset of PBAD subjects with high triglycerides and fasting FGF19 levels comparable to healthy individuals. The post prandial rise in FGF19 suggests no defect in the response of FGF19 synthesis in this subset. It may instead be caused by impaired BA absorption due to reduced ASBT expression which is also manifested as high serum triglycerides. PBAD may be a heterogenous condition with more than one underlying key abnormality.

Disclosure of Interest None Declared.

\section{OC-028 SMALL BOWEL CANCER IN THE UK}

${ }^{1} \mathrm{~L}$ Chan*, ${ }^{2} \mathrm{G}$ Evans. 'Gastroenterology, Manchester Royal Infirmary, Manchester, UK; ${ }^{2}$ Genetic Medicine, Manchester Royal Infirmary, Manchester, UK

\subsection{6/gutinl-2014-307263.28}

Introduction Small bowel cancer (SBC) is uncommon worldwide and accounts for only 5\% of all gastrointestinal (GI) malignancies despite the small bowel forming $75 \%$ of the GI tract. [1] Our understanding is limited by its rarity, insidious course, difficult assessment and late diagnosis, coupled with multiple histological subtypes. We aim to review the trend of SBC in the UK over the last two decades.

Methods The national UK Association of Cancer Registries (UKACR) database identified SBC patients diagnosed from January 1991 to January 2009. We retrospectively reviewed and carried out a descriptive analysis of SBC incidence rates with respect to gender, age, ethnicity (as per UK Census 2001) and socio-economic status (as per UK Indices of Deprivation 2004, 2007 and 2010) and mortality rates.

Results The registry identified 11,872 patients, 53.6\% male and $46.4 \%$ female, who were diagnosed at a singular peak mean age of 67 years over the study period. The overall incidence of SBC increased from 0.71 to 1.51 per 100,000 from 1991 to 2009 with mortality increasing simultaneously but to a lesser extent (Figure 1A). SBC was 1.5 times more common in males than females. They were most frequently located at the duodenum $(57.5 \%, \mathrm{n}=7860)$ where incidence almost tripled $(0.24$ to 0.63 per 100,000), and less frequently at the jejunum (12.1\%) and ileum (30.4\%) where incidence approximately doubled (0.07 to 0.11 and 0.14 to 0.33 per 100,000 respectively). The incidence in white patients was 1.5 times higher than black patients and 3 times higher than in Asian patients over the period 2001 to 2009 (Figure 1B). SBC incidence was unchanged with respect to socio-economic status.
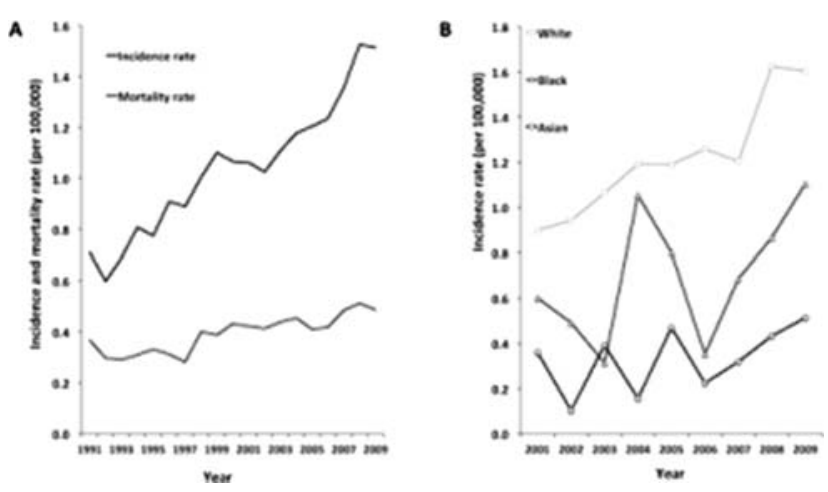

Abstract OC-028 Figure 1 (A) SBC incidence and mortality rates, 1991 to 2009. (B) SBC incidence by ethnic group, 2001 to 2009
Conclusion The incidence of SBC in the UK has increased over the last two decades with little improvement in mortality rates. It is most common in males in their $6^{\text {th }}$ decade and in the proximal small intestine, which is in keeping with current literature. However, the higher incidence in white patients is in contrast to the geographical variation seen in both United States SBC and UK colorectal cancer data. A more comprehensive understanding of the natural history, environmental and genetic predisposition is needed to allow for potential patient stratification, more efficient diagnosis and treatment and thus improving its poor prognosis.

\section{REFERENCE}

1 Ross et al. British Journal of Cancer. 1991;63:143-145

Disclosure of Interest None Declared.

\section{Liver section free papers}

\section{OC-029 RIFAXIMIN IS EFFICACIOUS IN THE TREATMENT OF CHRONIC OVERT HEPATIC ENCEPHALOPATHY: A UK LIVER MULTI-CENTRE EXPERIENCE}

${ }^{1} \mathrm{VC}$ Patel ${ }^{*},{ }^{2} \mathrm{~J}$ Orr, ${ }^{1} \mathrm{~J}$ Sturgeon, ${ }^{1} \mathrm{Z}$ Habtemariam, ${ }^{3} \mathrm{H}$ Preedy, ${ }^{4} \mathrm{P}$ Richardson, ${ }^{3} \mathrm{R}$ Aspinall, ${ }^{2} \mathrm{M}$ Hudson, 'DL Shawcross. 'Institute of Liver Studies, King's College Hospital NHS Foundation Trust, London, UK; ${ }^{2}$ Department of Hepatology, Freeman Hospital, Newcastle Hospitals NHS Foundation Trust, Newcastle, UK; ${ }^{3}$ Department of Gastroenterology and Hepatology, Queen Alexandra Hospital, Portsmouth Hospitals NHS Trust, Portsmouth, UK; ${ }^{4}$ Department of Hepatology, Royal Liverpool University Hospital, Liverpool, UK

\subsection{6/gutjnl-2014-307263.29}

Introduction Rifaximin- $\alpha$ is a non-absorbable antibiotic increasingly being used for the secondary prevention of recurrent overt hepatic encephalopathy (HE) in the UK. The therapeutic mechanism of rifaximin has yet to be elucidated, with reduction in gut ammonia production postulated. We undertook a UK multicentre retrospective audit of patients receiving rifaximin therapy for HE in 4 hospitals, two of which are liver transplant units, with the aim of assessing tolerability, impact on HE/liver disease severity and hospitalisation rates.

Methods Patient demographics, concurrent therapy, Child Pugh, MELD, UKELD and number of hospital admissions were collected 3 months prior to initiation of rifaximin therapy and then 3 months following treatment.

Results 170 patients were identified (mean age 57yrs \pm 12 ; 68\% male) over the period 05/2010-03/2013. Three month post treatment outcome data were available for 73 patients (43\%); 53 patients (31\%) died during the 3 month follow up period. Average duration of treatment was $79 \pm 121$ days, with therapy well tolerated in $97.6 \%$ of patients. $74 \%$ were taking concomitant lactulose with $23.5 \%$ on rifaximin monotherapy. No cases of Clostridium difficile infection were reported.

The most common aetiology was alcohol 90/170 (53\%) with $25(28 \%)$ actively drinking . 36 patients (21\%) were transplanted during the audit period. The predominant HE phenotype was episodic overt (67\%), with persistent overt featuring in $20 \%$ and the Parkinsonian phenotype in $6 \%$.

Admission data were available for $143 / 170$ (84\%) patients with a total of 444 admissions in the 3 months prior to therapy (average admission length $23 \pm 25$ days). The hospitalisation rate per patient fell significantly from $2.7 \pm 3.2$ to $1.0 \pm 1.8$ admissions in the 3 months following initiation of therapy $(\mathrm{p}<$ 